\title{
Effects of Combined and General Anesthesia on Cognitive Functions for Patients Undergoing Cardiac Surgery Under CPB
}

\author{
Greta Kasputytė, ${ }^{1}$ Rasa Bukauskienè ${ }^{1}$ Edmundas Širvinskas, ${ }^{2}$ Tadas Lenkutis, ${ }^{1}$ Renata Vimantaite,${ }^{3}$ \\ Judita Andrejaitiené \\ ${ }^{1}$ Department of Cardiac, Thoracic and Vascular Surgery, Lithuanian University of Health Sciences, Kaunas Clinics, Kaunas, Lithuania; \\ ${ }^{2}$ Institute of Cardiology, Lithuanian University of Health Sciences, Kaunas, Lithuania; ${ }^{3}$ Lithuanian University of Health Sciences, \\ Medical Academy, Faculty of Nursing, Kaunas, Lithuania
}

\section{ABSTRACT}

Background: Patients may experience a variety of neurological complications after heart surgery. The most common complication observed in clinical practice is delayed neurocognitive recovery (dNCR). The role of the anesthesiologist is very important, as the risk of dNCR may be reduced, depending on the anesthesia tactic chosen. Although the possibility that neuropsychological complications are less common in patients undergoing combined anesthesia (general + epidural) than in patients undergoing general anesthesia is not yet confirmed, the results are being discussed. The aim of this study was to determine impact of combined anesthesia (general + epidural) on cognitive functions of patients after cardiac surgery.

Methods: The prospective, case-controlled study included 80 patients undergoing cardiac surgery from 2015 to 2017 at the Department of Cardiothoracic and Vascular Surgery in the Hospital of Lithuanian University of Health Sciences Kauno Klinikos. After approval from the local bioethics center, informed consent was obtained from all study participants. Inclusion criteria were age 51 to 80 years, elective cardiac surgery, left ventricular ejection fraction $>35 \%$, anamnesis of not using agents affecting the central nervous system, absence of neuropathology, and sufficient renal function. Exclusion criteria were patients suffering from diseases causing cognitive function or using agents affecting the central nervous system, emergency or re-surgery, carotid artery atherosclerosis with artery diameter 50 or more percent reduction, and a patient's disagreement.

MMSE test and 6-CIT test were used for a cognitive function assessment, Trail making test and WAIS Digital Symbol Substitution test were used for psychomotor function assessment. All tests were used a day before surgery and seven days after surgery.

According to the planned anesthesia, patients were assigned into two groups: 1 - combined general + epidural anesthesia and 2 - general anesthesia. Standardized protocol of anesthesia was followed for all patients. Preoperative

Received April 11, 2021; accepted May 2, 2021.

Correspondence: Rasa Bukauskiene, Department of Cardiac, Thoracic and Vascular Surgery, Lithuanian University of Health Sciences, Kaunas Clinics, Kaunas, Lithuania. (e-mail: rasa.bukauskiene@lsmuni.lt) patients and surgery factors, preoperative and postoperative neuropsychological test results were recorded.

Results: Eighty patients were enrolled in the study. Both groups did not differ in demographic, perioperative values, and baseline (preoperative) test results. Postoperative (7th day) WAIS $(P=.042)$ and 6-item cognitive impairment $(P=.016)$ test results were statistically different when comparing the GA and CA groups. Comparing preoperative and postoperative test results, there was a significant decline in the WAIS test score in the GA group $(P=.013)$.

\section{INTRODUCTION}

Cardiovascular diseases are widespread around the world and, in Europe, a total of 1.68 million people died of cardiovascular pathologies in 2016. One of the most important treatments for myocardial revascularisation is coronary artery bypass graft $(\mathrm{CABG})$ surgery.

In 2018, 165,600 CABG operations were performed in 25 European Union countries, according to Eurostat. Lithuania ranks third in Europe, in terms of the number of operations performed, amounting to 55.6 per 100,000 inhabitants [Cardiovascular diseases statistics].

Patients may experience a variety of neurological complications after heart surgery. The most common complication observed in clinical practice is delayed neurocognitive recovery (dNCR). Delayed NCR is cognitive impairment, impacting memory, attention and concentration, which is temporally associated with anesthesia and surgery. Thirty percent to $80 \%$ of all patients, who have had heart surgery, display signs of dNCR, and this number is higher for patients receiving cardiac surgery with a cardiopulmonary bypass (CPB). Delayed NCR is associated with longer stays in the intensive care unit, infectious complications, longer hospitalization, poor cognitive and functional recovery, and mortality [Rundshagen 2014].

The exact etiopathogenesis of dNCR has not fully been clarified to date. In principle, the proper functioning of the central nervous system depends on the uptake of oxygen, carbon dioxide, food, and other substances; elimination of metabolic end-products; neurotransmitter balance; and other factors that may affect cerebral circulatory autoregulation [Wollman 1968; Blenkarn 1972]. An extremely high number 


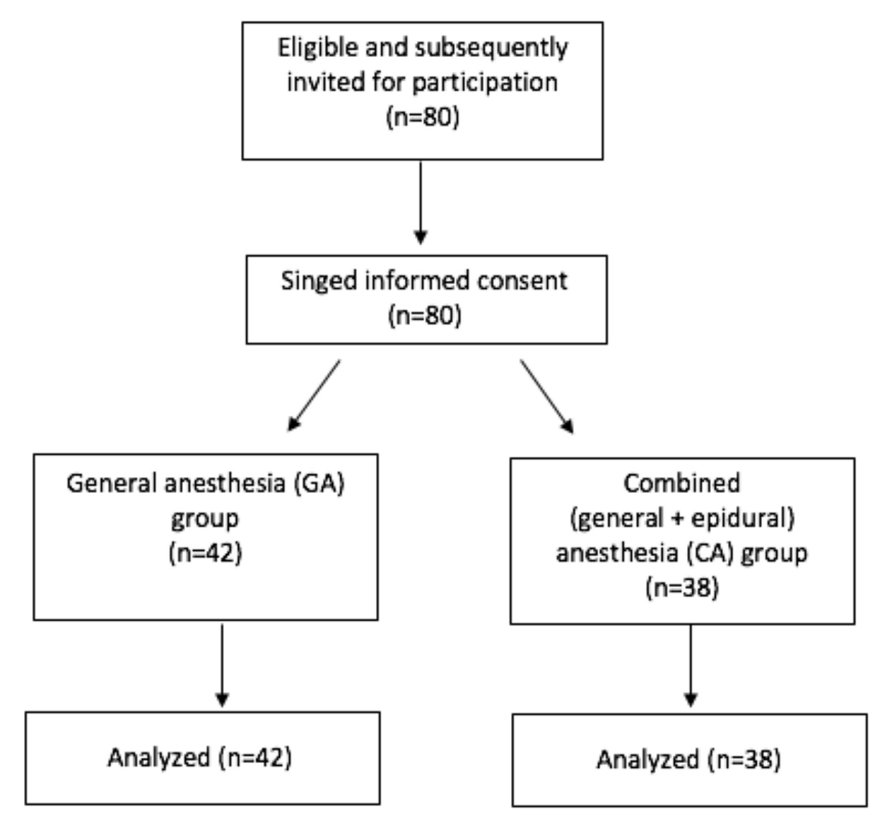

Figure 1. Flow chart for patient enrollment.

of dNCR risk factors are observed during cardiac surgeries: They may be related to the patient (concomitant vascular diseases, advanced age, arrhythmias, etc.), surgery and anesthesia (nature of surgery, CPB, duration of aortic clamping, embolization, anesthesia medications used, and opioid analgesics, etc.) or postoperative risk factors (hypotension, hyperglycemia, and $\mathrm{pH}$ imbalances, etc.) [Scolletta 2015].

The role of the anesthesiologist is very important, as the risk of dNCR may be reduced, depending on the anesthesia tactic chosen. Although the possibility that neuropsychological complications are less common in patients undergoing combined anesthesia (general + epidural) than in patients undergoing general anesthesia is not yet confirmed, the results are being discussed [Rasmussen 2003]. Therefore, in our study we have compared the impact of combined anesthesia (general + thoracic epidural) on the cognitive functions of patients after cardiac surgery.

\section{MATERIALS AND METHODS}

The prospective, case-control study took place at the Department of Cardiothoracic and Vascular Surgery in the Hospital of Lithuanian University of Health Sciences Kauno Klinikos between 2015 to 2017. After approval of the local ethics committee (BEC-MF-740) and with the patients' written consent, 80 patients undergoing CABG, mitral, aortic valve or combined on-pump cardiac surgery were enrolled in the study. The inclusion criteria were age 51 to 80 years, elective cardiac surgery, left ventricular ejection fraction $>35 \%$, anamnesis of not using agents affecting central nervous system, absence of neuropathology, and sufficient renal function. Exclusion criteria were patients suffering from diseases causing cognitive function or using agents affecting central nervous system, emergency or

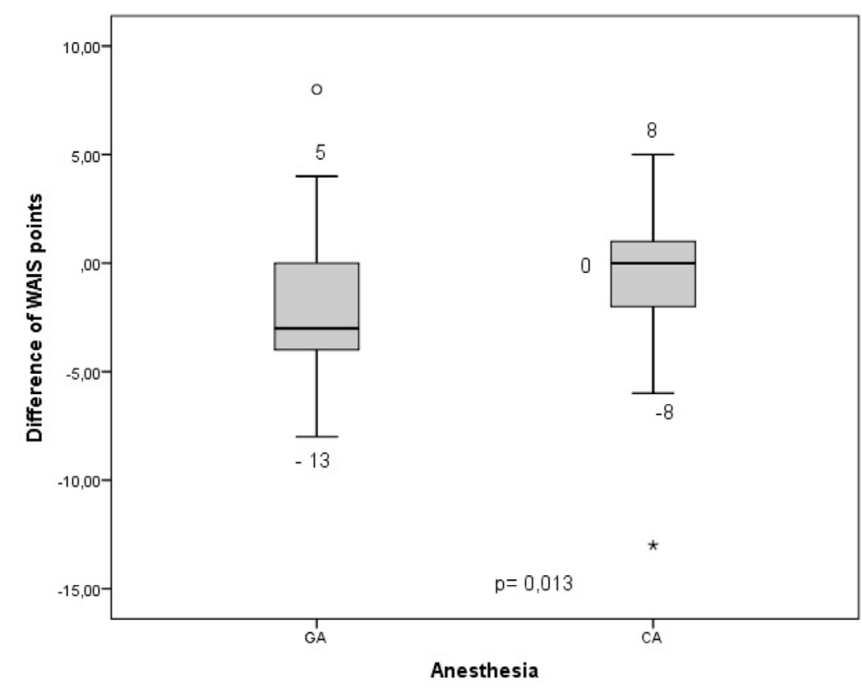

Figure 2. WAIS test scores difference pre- and postoperatively.

re-surgery, carotid artery atherosclerosis with artery diameter 50 or more percent reduction, and patient disagreement.

The patients randomly were assigned to receive a general or combined (general + epidural) anesthesia.

All patients perform neurocognitive evaluation test one day before surgery and seven days postoperatively. The tests were chosen to determine many different domains and included:

1. Minimal Mental State Examination (MMSE) administration of the test examines functions, including orientation, registration, attention and calculation, recall, language, and ability to follow simple commands [Crum].

2. Six-item cognitive impairment test (6 CIT) - as a screening test for dementia, comprises six questions about memory, calculation, and orientation. The 6 CIT correlates highly with MMSE [Abdel-Aziz 2015].

3. WAIS Digit symbol substitution test (WAIS) - is a subset of the Wechsler Intelligence Scale and primarily measures visual recording and includes a motor component. It provides perceptual reasoning, processing speed, verbal comprehension, and working memory. The test is scored by counting the number of correct symbols inserted [Jaeger 2018].

Anesthesia and surgery technique: An intravenous 16G cannula was inserted for fluid and drug therapy. A radial arterial 20G cannula was inserted under local anesthesia for invasive arterial blood pressure monitoring. Patients, who allocated into combined anesthesia group, were positioned in the sitting decubitus position. An epidural catheter was inserted in the thoracic T2-T3 intervertebral space level, 5 $\mathrm{cm}$ in the cranial direction. Lidocaine $2 \% 3 \mathrm{ml}$ test dose was injected via an epidural catheter, followed by $6 \mathrm{ml}$ bolus dose of $0.5 \%$ bupivacaine. An intravenous fluid load of $6-7 \mathrm{ml} / \mathrm{kg}$. of Ringer's acetate was infused to all patients before anesthesia induction.

For all patients, anesthesia was induced after preoxygenation by face mask with $100 \%$ oxygen with intravenous (i.v.) fentanyl 1-2 $\mu \mathrm{g} / \mathrm{kg}$, i.v. propofol $2 \mathrm{mg} / \mathrm{kg}$, and i.v. 
Table 1. Characteristics of study population

\begin{tabular}{lccc}
\hline Variables & $\begin{array}{c}\text { GA group } \\
(\mathrm{N}=42)\end{array}$ & $\begin{array}{c}\text { CA group } \\
(\mathrm{N}=38)\end{array}$ & $P$ \\
\hline Age (years, mean ( $\pm \mathrm{SD})$ & $67.2( \pm 7.3)$ & $64.9( \pm 7.4)$ & .167 \\
Male, $\mathrm{n}(\%)$ & $22(52.4)$ & $25(65.8)$ & .224 \\
Female, $\mathrm{n}(\%)$ & $20(47.6)$ & $13(34.2)$ & \\
Diabetes mellitus (\%) & $11(26.2)$ & $9(23.7)$ & .796 \\
$\begin{array}{l}\text { Chronic atrial fibrillation (\%) } \\
\text { Duration of CPB (min, mean }( \pm \mathrm{SD})\end{array}$ & $84.6( \pm 16.3)$ & $87.7( \pm 21.7)$ & .547 \\
$\begin{array}{l}\text { Duration of aortic cross-clamp (min, } \\
\text { mean }( \pm \text { SD) }\end{array}$ & $41.9( \pm 13.5)$ & $48.9( \pm 19.6)$ & .063 \\
$\begin{array}{l}\text { Length of stay in ICU (hours, mean } \\
( \pm \text { SD) }\end{array}$ & $68.6( \pm 30.3)$ & $70.4( \pm 27.9)$ & .6
\end{tabular}

rocuronium $0.6 \mathrm{mg} / \mathrm{kg}$. After tracheal intubation, lungs were ventilated with a supply of oxygen/air $50-60 \%$ in the pressure control mode. Anesthesia was maintained with sevoflurane 0.8-1.0 MAC. As an analgesic component in the general anesthesia (GA) group, i.v. fentanyl 10-12 $\mu \mathrm{g} / \mathrm{kg}$ was used and in the combined anesthesia (CA) group, epidural continuous infusion of $0.125 \%$ bupivacaine and fentanyl $5 \mu \mathrm{g} / \mathrm{ml}$ mixture was used, based on vital signs according to judgement of an experienced anesthesiologist (to individual patient's response).

Middle sternotomy section and standard technique for anastomosis formation or valve surgery was performed. After systemic heparinization and cannulation, CPB was established using a roller pump "Stockert III" and hollow-fiber membranous oxygenator "Compactflo D703 (Dideco, Mirandola, Italy). The CPB circuit was primed with $1200-1500 \mathrm{ml}$ of Ringer's acetate crystalloid solution and 10000IU of heparin. After full CPM support, pump flow was maintained at a cardiac index of $2.41 / \mathrm{min} / \mathrm{m} 2$. Cardiac arrest was achieved with cold hypercalemic St. Thomas, according to clinical protocol.

Standard monitoring of vital signs consisted of an electrocardiogram, invasive arterial blood pressure, heart rate, pulse oximetry, esophageal and body core temperature, central venous blood pressure, and urine output.

Depth of anesthesia was monitored, and BIS maintained from the 40 to 60 range. Mean arterial pressure was maintained between 50 and $75 \mathrm{mmH}$ by infusion of intravenous fluids and administration of sympathomimetics, if needed. Normothermia and normocapnia was maintained during surgery.

All patients were transferred to the ICU after surgery and treated, according to ICU protocols.

In the GA group, postoperative pain was treated with boluses of i.v. morphine until $10 \mathrm{mg}$ on demand; in the CA group, pain was relief by the same epidural infusion with the bupivacaine and fentanyl mixture, which was maintained at least 24 hours. Patients in both groups received i.v. acetaminophen $1000 \mathrm{mg}$ four times a day during two postoperative days.

Statistical analysis was performed using SPSS 22.0. Quantitative variables were described as mean \pm standard
Table 2. Characteristics of preoperative test scores

\begin{tabular}{lccc}
\hline Variables & $\begin{array}{c}\text { GA group } \\
(\mathrm{N}=42)\end{array}$ & $\begin{array}{c}\text { CA group } \\
(\mathrm{N}=38)\end{array}$ & $P$ \\
\hline $\begin{array}{l}\text { WAIS score (mean }( \pm \mathrm{SD}) \\
\text { MMSE score }\end{array}$ & $19.33( \pm 7.11)$ & $20.89( \pm 6.34)$ & .305 \\
$\quad$ Median & 28 & 28 & \\
Min & 18 & 22 & .447 \\
Max & 30 & 30 & \\
Six-item score & & & \\
Median & 2 & 2 & \\
Min & 0 & 0 & .326 \\
Max & 18 & 16 &
\end{tabular}

deviation (SD). The Kolmogorov-Smirnov test was used to determine data distribution. Student's t-test, paired samples t-test was used for normal distribution. Mann-Whitney U-test was used for non-normally distributed variables. The difference was considered to be significant when $P$ value was $<.05$.

\section{RESULTS}

Eighty patients were enrolled in the study (Figure 1).

Patient demographic and perioperative data are shown in Table 1 . There were no differences in sex, age, diabetes mellitus, chronic atrial fibrillation, duration of $\mathrm{CPB}$ and aortic cross-clamp, and length of stay in the ICU between the two groups.

All preoperative (baseline) test results did not differ statistically significantly among the GA and CA groups (Table 2).

Postoperative (day 7) WAIS and 6-item cognitive impairment test results were statistically different, comparing the GA and CA groups (Table 3).

Comparing preoperative and postoperative test results, there was a significant decline in WAIS test score in the GA group (Figure 2).

\section{DISCUSSION}

The nomenclature of postoperative cognitive declines changed in 2018. It is recommended to use the term "postoperative delirium" or "postoperative neurocognitive recovery" to postoperative cognitive impairment, which emerges from immediately after postoperative period and lasts up to 30 days.

Assessment of cognitive functions is one of the most relevant tasks in research. It is recommended to choose cognitive tests that cover as many cognitive functions as possible - memory, attention, and psychomotor speed functions tend to be impaired after cardiac surgery. Select tests should not be repeated with each other to minimize disruption in the early postoperative periods [Polunina 2014]. 
Table 3. Characteristics of postoperative test scores

\begin{tabular}{lccc}
\hline Variables & $\begin{array}{c}\text { GA group } \\
(\mathrm{N}=42)\end{array}$ & $\begin{array}{c}\text { CA group } \\
(\mathrm{N}=38)\end{array}$ & $P$ \\
\hline WAIS score (mean $( \pm \mathrm{SD})$ & $17.14( \pm 6.48)$ & $20.03( \pm 5.97)$ & .042 \\
MMSE score & & & \\
Median & 28 & 28 & \\
Min & 18 & 23 & .096 \\
Max & 30 & 30 & \\
Six-item score & & & \\
Median & 4 & 2 & \\
Min & 0 & 0 & .016 \\
Max & 18 & 14 &
\end{tabular}

Mostly, patients perform five to 10 tests to assess cognitive function in a number of studies investigating dNCR. However, it takes more than 30 minutes to perform those tests, so patients get tired and may stop concentrating. Another difficulty is retesting, which can have a learning effect, when patients memorize a task and perform them better the second time [Gregory 2006]. Therefore, three different and short tests - MMSE, 6-item and WAIS - were elected in our study.

Patients performed each test twice: a day before surgery and seven days after. It should be noted that steady or improved test results might not reflect an improvement in the patient's cognitive abilities. It could be that the choice of anesthetic technique was associated neither with a significant difference in overall scores, nor with a significant difference in scores at any given time. Therefore, a small sample of patients and unwillingness to cooperate with researchers may have affected test results.

We have attempted to determine and compare the incidence of dNCR between general and combined anesthesia groups of patients undergoing cardiac surgery. Neurocognitive function tests' results did not significantly differ among the groups after the surgery, except 6-item cognitive impairment and WAIS test results were worse in the general anesthesia group. Comparing 6-item test results between the groups, we noticed increased risk of dementia in the general anesthesia group. Interestingly, we found that WAIS test results significantly were lower after surgery than before in the general anesthesia group. As WAIS tests are sensitive to brain injury, memory and processing speed, these findings could show that memory and processing speed capabilities decline after surgery in the general anesthesia group [Rasmussen 2003; Weber 2009]. However, according to our results, lower doses of narcotic analgesics used in the combined anesthesia group may decrease the frequency of neurocognitive dysfunction in comparison with the general anesthesia group [Rasmussen 2003; Weber 2009; Mason 2010; Evered 2011]. Also, comparing both anesthesia groups, tests tend to be better in the combined anesthesia group, but a bigger plot is needed to ensure significant results.
Despite the fact that postoperative cognitive changes were first reported in 1955, the results of other studies still are controversial [Bedford 1955]. According to an ISPOCD2 trial, the incidence of dNCR seven days after major non-cardiac surgery was higher in the general anesthesia group, comparing with regional anesthesia [Rasmussen 2003]. Comparing GALA's study trial test results, neurocognitive performance decreased in the general anesthesia group for patients after carotid endarterectomy [Weber 2009]. On the other hand, according to Mason et al.'s conducted metaanalysis results, general anesthesia was marginally insignificantly associated with dNCR [Mason 2010]. In our study, we found only memory and processing speed capabilities decline by using the WAIS test in the general anesthesia group, and there was no significant decline in the combined anesthesia group before and after surgery. However, we should take into account that previous studies analyzed only regional anesthesia, as we included a combined (general + regional) anesthesia into our study.

Other research highlights chosen time points after surgery. It was noticed that additional cognitive improvement occurred in patients who were tested more than one week or three months after surgery, resulting in an underestimation of the prevalence of dNCR [Evered 2011; Monk 2008]. Selnes et al suggest that change in neurocognitive function after CABG is associated with medical and surgical variables and the specifics of these associations depend on the choice of time points after surgery [Selnes 1999]. We tested patients one time (seven days) after surgery. According to other studies, the incidence of dNCR is higher seven days after surgery, comparing with three months after surgery. This suggests that in our study, the decline of neurocognitive functions could be underestimated taking only one time point after surgery.

We did not investigate the pain scores after surgery. According to protocol that exists in our department, we maintain a pain score of less than 3 points. So, patients get as many analgesics as is enough. On the other hand, these medications could influence cognitive function, and it is possible these factors may lead to poorer outcomes. So, one area that requires further examination is the possibility that another medication (such as analgesics) and/or comorbidities may lead to poorer neuropsychological performance [Cullen 2007]. We did not investigate other medications' influence on cognitive functions. But it is possible that these factors may lead to worse outcomes.

Our study had several limitations. There is not a standardized questionnaire for evaluating factors that influence dNCR, which is suitable for multiple comparisons and is not sensitive to learning effect. Moreover, we included a small sample of patients. Hence, this study could be expanded to include a bigger sample, and results would be more reliable by using standardized questionnaires.

\section{CONCLUSION}

According to our research, general anesthesia is associated with dNCR, comparing with combined anesthesia. Also, there is a tendency that memory and processing speed capabilities decrease after general anesthesia and CA may be of the component for dNCR prevention. 


\section{REFERENCES}

Abdel-Aziz K, Larner AJ. 2015. Six-item cognitive impairment test (6CIT): pragmatic diagnostic accuracy study for dementia and MCI. Int Psychogeriatr. Jun;27(6):991-7.

Bedford PD. 1955. Adverse cerebral effects of anaesthesia on old people. Lancet. 259-263.

Blenkarn GD, Briggs G, Bell J, Sugioka K. 1972. Cognitive Function after Hypocapnic Hyperventilation. Anaesthesiology. 37(4):381-6.

Cardiovascular diseases statistics. Available from https:// ec.europa.eu/eurostat/statistics-explained/index.php/ Cardiovascular_diseases_statistics\#Cardiovascular_healthcare

Crum RM, Anthony JC, Bassett SS, Folstein MF. Population-based norms for the Mini-Mental State Examination by age and educational level. JAMA 269: 2386-2391.

Cullen B, O'Neill B, Evans JJ, Coen RF, Lawlor BA. 2007. A review of screening tests for cognitive impairment. J Neurol Neurosurg Psychiatry. Aug; 78(8): 790-799.

Evered L, Scott D, Silbert B, Maruff. 2011. Postoperative Cognitive Dysfunction Is Independent of Type of Surgery and Anesthetic. Anesthesia \& Analgesia. 112(5):1179-1185.

Gregory L, Anna W. 2006. Evidence-based clinical update: General anesthesia and the risk of delirium and postoperative cognitive dysfunction. General anesthesia. July; 53: 669677.

Jaeger J. 2018. Digit Symbol Substitution Test: The Case for Sensitivity Over Specificity in Neuropsychological Testing. J Clin Psychopharmacol. Oct;38(5):513-519.

Mason SE, Noel-Storr A, Ritchie CW. 2010. The impact of general and regional anesthesia on the incidence of post-operative cognitive dysfunction and post-operative delirium: a systematic review with meta-analysis. J Alzheimers Dis. 22(3):67-79.
Monk T, Weldon C, Garvan C, Dede D, Van der Aa M, Heilman K, et al. 2008. Predictors of Cognitive Dysfunction after Major Noncardiac Surgery. Anesthesiology. 108:18-30.

Polunina AG, Golukhova EZ, Guekht AB, Lefterova NP, Bokeria LA. 2014. Cognitive Dysfunction after On-Pump Operations: Neuropsycho $\neg$ logical Characteristics and Optimal Core Battery of Tests. Stroke Res Treat. 1-18.

Rasmussen LS, Johnson T, Kuipers HM, Kristensen D, Siersma VD, Vila P, et al. 2003. Does anaesthesia cause postoperative cognitive dysfunction? A randomised study of regional versus general anaesthesia in 438 elderly patients. Acta Anaesth Scand. 47(3): 260-6.

Rundshagen I. 2014. Postoperative kognitive Dysfunktion. Dtsch. Arztebl. Int. 111 (8), 119-125.

Selnes OA, Goldsborough MA, Borowicz LM Jr, Enger C, Quaskey SA, McKhann GM. 1999. Determinants of cognitive change after coronary artery bypass surgery: a multifactorial problem. The Annals of Thoracic Surgery. 67(6):1669-1676.

Scolletta S, Taccone FS, Donadello K. 2015. Brain injury after cardiac surgery. Minerva Anestesiologica. 81:662-677.

Weber CF, Friedl H, Hueppe M, Hintereder G, Schmitz-Rixen T, Zwissler B, Meininger D. 2009. Impact of general versus local anesthesia on early postoperative cognitive dysfunction following carotid endarterectomy: GALA Study Subgroup Analysis. World J Surg. Jul;33(7).

Wollman SB, Orkin LR. 1968. Postoperative human reaction time and hypocarbia during anaesthesia. Br J Anaesth. 40(12):920-6. 